\title{
A Young Man with Non-alcoholic Steatohepatitis and Serum Anti-mitochondrial Antibody Positivity
}

\author{
Takuya Seike ${ }^{1,2}$, Takuya Komura ${ }^{1}$, Yoshiaki Shimizu ${ }^{1}$, Hitoshi Omura ${ }^{1}$, Tatsuo Kumai ${ }^{1}$, \\ Takashi Kagaya ${ }^{1}$, Hajime Ohta ${ }^{1}$, Atsuhiro Kawashima ${ }^{3}$, Kenichi Harada ${ }^{4}$, \\ Shuichi Kaneko ${ }^{2}$ and Masashi Unoura ${ }^{1}$
}

\begin{abstract}
:
A 37-year-old obese man who was a social drinker was admitted to our hospital to undergo a detailed examination for liver injury with anti-mitochondrial antibody positivity. Abdominal ultrasonography revealed moderate fatty liver. A histological analysis showed steatosis of approximately $30 \%$ of the hepatocytes, focal necrosis, a few ballooning hepatocytes and lobular inflammation suggestive of steatohepatitis, epithelioid granuloma and irregularity of the sequence of the bile duct epithelium accompanied by lymphocyte infiltration suggestive of chronic cholangitis. He was diagnosed with non-alcoholic steatohepatitis complicated with primary biliary cholangitis. His liver injury was improved by weight loss and high-dose ursodeoxycholic acid treatment.
\end{abstract}

Key words: non-alcoholic steatohepatitis, anti-mitochondrial antibody, chronic cholangitis, primary biliary cholangitis, ursodeoxycholic acid

(Intern Med 57: 3093-3097, 2018)

(DOI: 10.2169/internalmedicine.0405-17)

\section{Introduction}

The characteristic histological findings of non-alcoholic steatohepatitis (NASH) are steatosis, ballooning and lobular inflammation, and they typically develop around the central veins $(1,2)$. However, bile duct lesions, such as cholangitis, are generally uncommon in NASH patients (1).

The presence of anti-mitochondrial antibodies (AMAs) in serum shows high sensitivity and specificity for the diagnosis of primary biliary cholangitis (PBC) in the context of unexplained bile duct lesions (3). However, AMAs are sometimes detected in patients without $\mathrm{PBC}$, and there have been a few reports of AMA positivity in NASH patients (4-7).

We herein report a young, obese man with NASH accompanied by AMA positivity and histological chronic cholangitis.

\section{Case Report}

A 37-year-old man who underwent percutaneous coronary intervention for acute myocardial infarction at 34 years of age presented to our hospital with liver injury. He had adultonset hypertension, dyslipidemia, diabetes and sleep apnea syndrome. He had taken rosuvastatin calcium, enalapril maleate, metformin hydrochloride, carvedilol, nicorandil, aspirin and esomeprazole. He had smoked 1 pack of cigarettes per day for over 22 years. He was a social drinker.

He was admitted to our hospital for the evaluation of liver injury. His weight on admission was $95 \mathrm{~kg}$ (74 kg at 20 years of age), and his body mass index was $37.5 \mathrm{~kg} / \mathrm{m}^{2}$. There were no significant findings on a physical examination. Laboratory tests revealed that his serum aminotransferase and $\gamma$-glutamyltransferase $(\gamma$-GTP) levels were increased, but his serum alkaline phosphatase level was nor-

\footnotetext{
${ }^{1}$ Department of Gastroenterology, National Hospital Organization Kanazawa Medical Center, Japan, ${ }^{2}$ System Biology, Graduate School of Advanced Preventive Medical Science, Kanazawa University, Japan, ${ }^{3}$ Department of Clinical Laboratory, National Hospital Organization Kanazawa Medical Center, Japan and ${ }^{4}$ Department of Human Pathology, Kanazawa University Graduate School of Medical Science, Japan Received: October 24, 2017; Accepted: February 18, 2018; Advance Publication by J-STAGE: June 6, 2018 Correspondence to Dr. Takuya Seike, verdauungsapparat@yahoo.co.jp
} 
Table 1. Biochemical Parameters.

\begin{tabular}{lcc}
\hline \multicolumn{1}{c}{ Examination parameters } & Reference value & $\begin{array}{c}\text { Value } \\
\text { admission }\end{array}$ \\
\hline White blood cell count $\left(\times 10^{3} / \mathrm{mm}^{3}\right)$ & $4.5-9.0$ & 9.4 \\
Hemoglobin level (g/dL) & $13.0-17.1$ & 15 \\
Platelet level (×103/mm $\left.{ }^{3}\right)$ & $150-350$ & 232 \\
Aspartate transaminase level (U/L) & $13-33$ & 72 \\
Alanine aminotransferase level (U/L) & $8-42$ & 133 \\
Alkaline phosphatase level (U/L) & $115-359$ & 196 \\
$\gamma$-Glutamyltransferase level (U/L) & $10-47$ & 142 \\
Total bilirubin level (mg/dL) & $0.3-1.2$ & 0.3 \\
Ureic acid level (mg/dL) & $3.6-7.0$ & 6.3 \\
C-reactive protein level (mg/dL) & $0.00-0.40$ & 0.77 \\
Alubumin level (g/dL) & $4.0-5.0$ & 3.9 \\
Prothrombin activity (\%) & $70-130$ & 99 \\
Total cholesterol level (mg/dL) & $128-219$ & 137 \\
Triglyceride level (mg/dL) & $30-149$ & 137 \\
Hemoglobin A1c level (\%) & $4.6-6.2$ & 6.2 \\
Immunoglobulin G level (mg/dL) & $870-1,700$ & 1,582 \\
Immunoglobulin A level (mg/dL) & $110-410$ & 439 \\
Immunoglobulin M level (mg/dL) & $35-220$ & 222 \\
Antinuclear antibody & $<1: 40$ & 40 \\
Antimitochondrial antibody & $<1: 20$ & 80 \\
Antimitochondrial M2 antibody (U/mL) & $0-7.0$ & 400 \\
Antismooth muscle antibody & $<1: 40$ & 20 \\
Hyaluronic acid level (ng/mL) & $0-50$ & 100 \\
Type IV collagen 7s domain level (ng/mL) & $0-5-0.8$ \\
Type III procollagen-N-peptide (U/L) & & 5.5 \\
\hline & &
\end{tabular}

mal. The serum immunoglobulin $\mathrm{M}$ and AMA-M2 levels were $222 \mathrm{mg} / \mathrm{dL}$ and $400 \mathrm{U} / \mathrm{mL}$, respectively, and his AMA titer was 1:80 (Table 1). Markers for hepatitis B virus (HBV) and hepatitis $\mathrm{C}$ virus (HCV) were both negative. The thyroid hormone level was normal. Tests for antithyroglobulin antibody, anti-thyroperoxidase antibody, anticyclic citrullinated peptide antibody, rheumatoid factor, antiSjögren's syndrome A antibody and anti-Sjögren's syndrome $\mathrm{B}$ antibody were negative.

Ultrasonography showed liver deformity, ultrasound attenuation, decreased detail of intra-hepatic vascular architecture and exaggerated differences between the kidney parenchyma and liver echogenicity. Computed tomography showed the appearance of irregular surfaces and dull edges with mild splenomegaly. There was no ascites or hepatocellular carcinoma (Fig. 1). Upper gastrointestinal endoscopy revealed no esophageal varices as a manifestation of portal hypertension. A histological analysis of a liver biopsy showed steatosis of approximately 30\% of hepatocytes, many with focal necrosis or apoptosis accompanied by a few ballooning hepatocytes and lobular inflammation suggestive of steatohepatitis, as well as epithelioid granuloma and irregularity of the sequence of the bile duct epithelium accompanied by lymphocyte infiltration around the damaged bile duct, suggestive of chronic cholangitis (Fig. 2). Chronic non-suppurative destructive cholangitis (CNSDC) and bile duct loss were not apparent. Given these findings, we diag- nosed the patient with NASH complicated with PBC (steatosis, grade 1; lobular inflammation, grade 2; ballooning, grade 1 , NAFLD activity score 4 , i.e., NASH probable, according to the NASH Clinical Research Network scoring system, NASH grade 2/stage 2, according to the Brunt classification system, together with $\mathrm{PBC}$ stage 2; bile duct loss, 0 ; fibrosis, 1; orcein-positive granule, 0/cholangitis activity, 2/hepatitis activity, 2/according to Nakanuma's criteria).

The patient's clinical course is shown in Fig. 3. We educated him strictly regarding the need for weight loss and improvements in his lifestyle. However, even after such counseling his weight continued to increase, and his serum aminotransferase and $\gamma$-GTP levels had increased as well. We prescribed ursodeoxycholic acid (UDCA) at $600 \mathrm{mg}$ per day due to his exacerbated bile duct lesion and repeatedly emphasized the importance of weight loss. The decrease in the serum aminotransferase level with his eventual body weight loss was remarkable, but his serum $\gamma$-GTP level remained high. Increasing the UDCA dose to $900 \mathrm{mg}$ reduced his serum $\gamma$-GTP level.

\section{Discussion}

We report here the case of a young, obese man with NASH accompanied by AMA positivity and histological chronic cholangitis that was diagnosed as NASH complicated with PBC. 

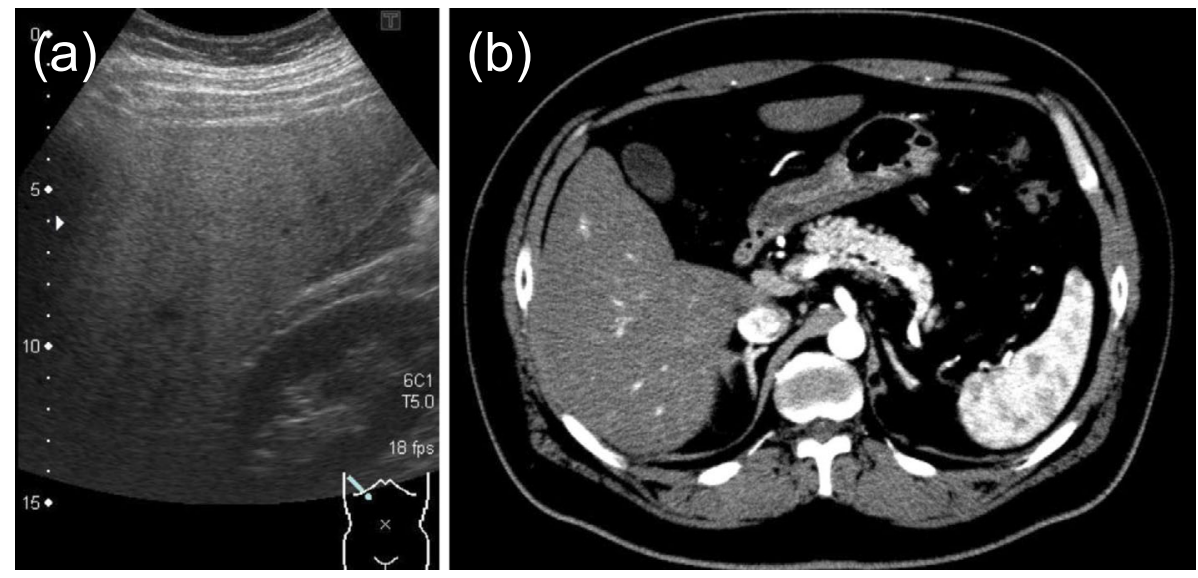

Figure 1. (a) Ultrasonography showed liver deformity, ultrasound attenuation, decreasing detail of the intra-hepatic vascular architecture and exaggeration of differences between kidney parenchyma and liver echogenicity. (b) Contrast-enhanced computed tomography of the abdomen shows a liver deformity with splenomegaly, indicating chronic liver disease. No hepatocellular carcinoma was evident.
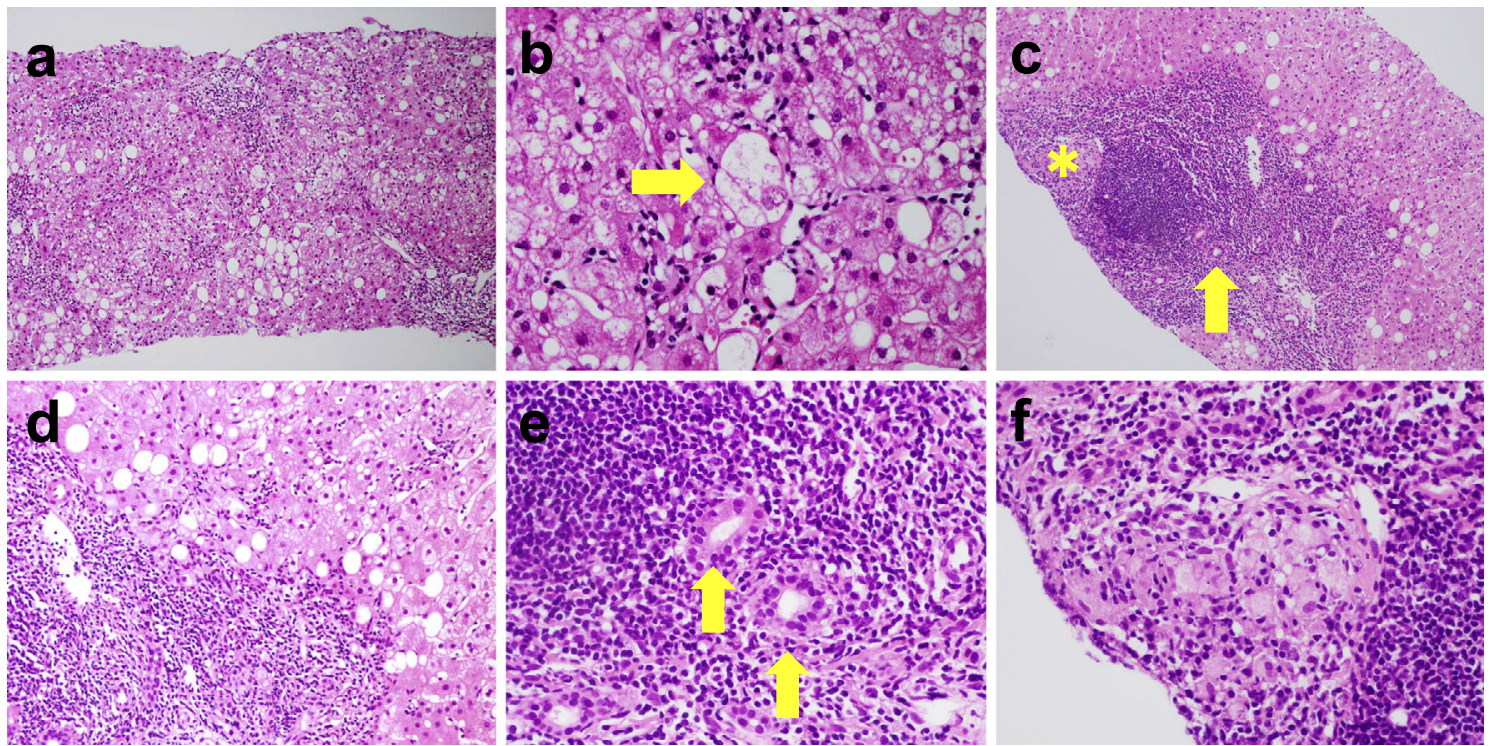

Figure 2. Histology of liver biopsy specimens. (a) Hematoxylin and Eosin (H\&E) staining (×10). The hepatic lobule structure was largely maintained, but cellular and fibrous expansion of the portal tract was notable, and partial progression of the fibrous partition was seen. Focal necrosis was seen in the hepatic parenchyma. Approximately 30\% of the area showed steatosis. (b) H\&E staining (×100). Ballooning hepatocytes were observed (arrow). (steatosis, grade 1; lobular inflammation, grade 2; ballooning, grade 1, NAFLD activity score 4, i.e., NASH probable, according to the NASH Clinical Research Network scoring system, NASH grade 2/stage 2, according to the Brunt classification system). (c) H\&E staining (×10). Chronic cholangitis (arrow), granuloma (*) and lymph folliclelike cell accumulation with the germinal center were seen in portal area into which lymphocytes had severely infiltrated. (d) H\&E staining $(\times 100)$. Interface hepatitis with lymphocytic infiltration was seen. (e) H\&E staining $(\times 100)$. A high-power-field view of the part indicated by an arrow in (c) is shown. Irregularity of the sequence of the bile duct epithelium was seen (arrow), suggesting chronic cholangitis. (f) $\mathrm{H} \& \mathrm{E}$ staining $(\times 100)$. A high-power-field view of the part indicated by an asterisk in (c) is shown. The accumulation of epithelioid cells accompanied by lymphocytic infiltration and fibrosis were seen, suggesting epithelial granuloma. (PBC stage 2; bile duct loss, 0; fibrosis, 1; orcein-positive granule, 0 /cholangitis activity, 2/hepatitis activity, 2/according to Nakanuma's criteria). 


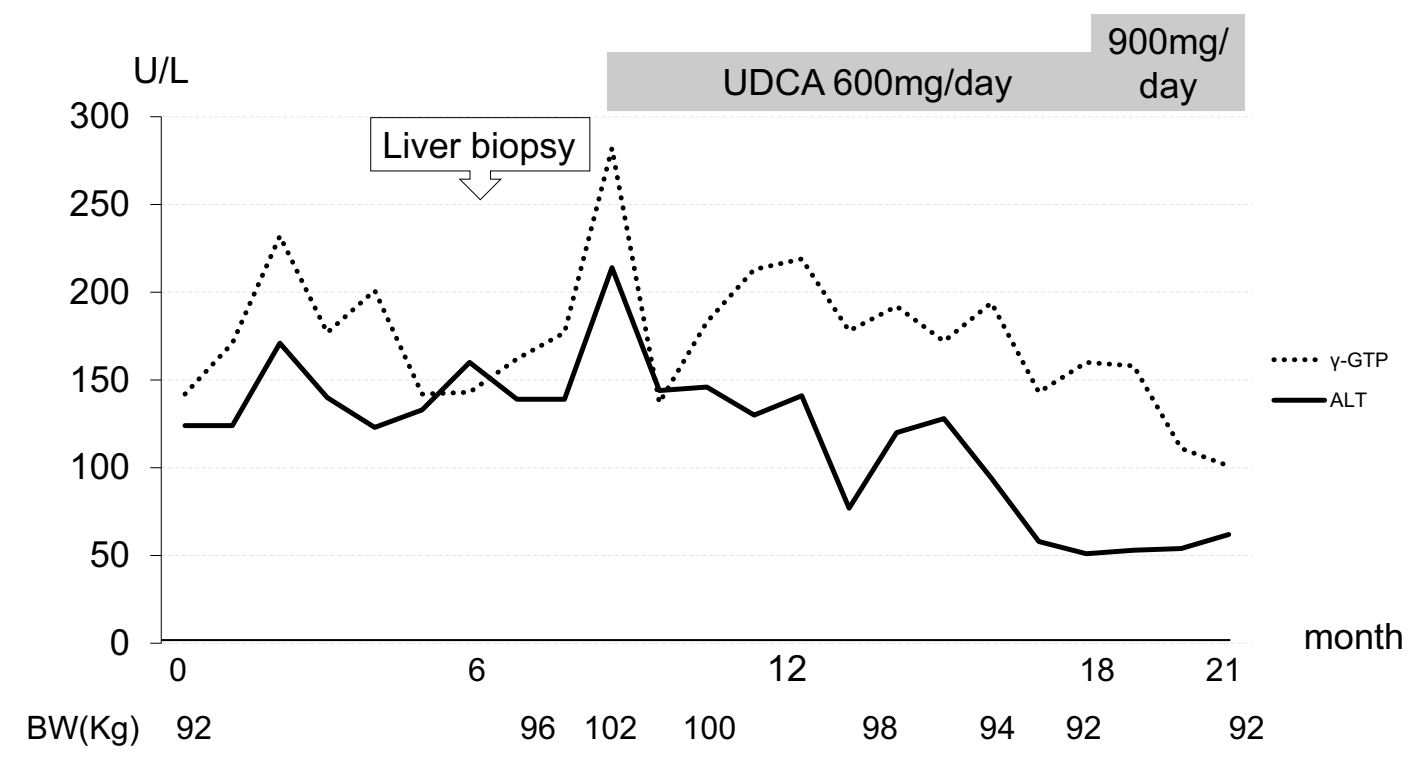

Figure 3. Clinical course of the patient.

Table 2. The Frequency of AMA Positivity in Non-PBC Patients.

\begin{tabular}{|c|c|c|c|}
\hline References & Subject & Patients n. & AMA positivity $\mathrm{n}(\%)$ \\
\hline 10 & general population & 1,714 & $11(0.64 \%)$ \\
\hline 4 & steatohepatitis-related liver disease (NAFLD or ALD) & 398 & $4(1 \%)$ \\
\hline 5 & NAFLD & 84 & $2(2.4 \%)$ \\
\hline 6 & NAFLD/NASH & $1,266 / 404$ & $50(4 \%) / 32(8 \%)$ \\
\hline 7 & NAFLD & 100 & $2(2 \%)^{*}, 10(10 \%) \dagger$ \\
\hline 11 & $\mathrm{CHB}$ & 190 & $0(0 \%)$ \\
\hline 12 & $\mathrm{CHB} / \mathrm{CHC}$ & $325 / 71$ & $22(6.8 \%) / 2(2.8 \%)$ \\
\hline 13 & $\mathrm{CHC}$ & 237 & $18(8 \%)$ \\
\hline 14 & AIH & 41 & $14(34 \%)$ \\
\hline 15 & Acute liver failure & 69 & $28(40.6 \%)$ \\
\hline
\end{tabular}

* detected by indirect immunofluorescence on rat tissues. $\dagger$ detected by indirect immunofluorescence on Hep- 2 cells. NAFLD: non-alcoholic fatty liver disease, ALD: alcoholic liver diesase, NASH: non-alcoholic steatohepatitis, CHB: chronic hepatitis $\mathrm{B}, \mathrm{CHC}$ : chronic hepatitis $\mathrm{C}, \mathrm{AIH}$ : autoimmune hepatitis

When two diseases coexist, it can be difficult to determine whether or not the pathologies of the diseases are related. If the pathologies of NASH and PBC are not related, patients with $\mathrm{PBC}$ can be complicated with NASH as a lifestyle-related disease. This is what happened in the present case, as the patient was accidentally diagnosed with PBC during a detailed examination of liver injury actually caused by NASH. If the pathologies of NASH and PBC are related, one can trigger the other. However, $\mathrm{PBC}$ acts protectively against metabolic syndrome in some cases (8) and does not usually cause hepatic steatosis. As such, PBC is unlikely to induce NASH. Therefore, based on the assumption that these pathologies were related, we considered that NASH may have contributed to or accelerated the onset of PBC.

In patients with $\mathrm{PBC}$, which is characterized histologically by bile duct lesions and chronic cholangitis, AMA positivity (a diagnostic criterion of $\mathrm{PBC}$ ) is present in around $95 \%$ of patients (9). In contrast, in non-PBC pa- tients, the frequency of AMA positivity in the general population is $0.64 \%(10)$, whereas that in patients with liver injury [chronic hepatitis B $(11,12)$, chronic hepatitis C (13), autoimmune hepatitis (14), non-alcoholic fatty liver disease (NAFLD) (4-7), and acute liver failure (15)] is even higher (Table 2). Therefore, in non-PBC patients with factors predisposing them to eventually develop $\mathrm{PBC}$, liver injury might trigger AMA production or contribute to the manifestation of AMAs below detection sensitivity. An example of such a predisposing factor is the pre-existing infection of Escherichia coli. AMA targets the E2 subunit of the pyruvate dehydrogenase complex (PDC-E2) (3) and exhibits molecular mimicry and immunological cross-reactivity with $E$. coli (16). Immunological cross-reactivity caused by liver injury in NASH may contribute to AMA production and pathogenesis of bile duct lesions, i.e., the onset of PBC in patients infected with bacteria that exhibit molecular mimicry with PDC-E2. In the present case, the patient had been obese since his 20s, raising the possibility that NASH pre- 
ceded PBC. Therefore, liver injury induced by NASH might contribute to or accelerate the onset of $\mathrm{PBC}$ in patients predisposed to PBC.

The mainstay treatments for patients with NAFLD are body weight control and lifestyle improvements (17). UDCA is the only approved drug for the treatment of $\mathrm{PBC}$, and it not only improves biochemical abnormalities but also delays histological progression (9). However, the widespread use of UDCA in NAFLD patients cannot be recommended due to insufficient evidence (18). In this case, UDCA treatment was started because of an increased $\gamma$-GTP level during temporarily aggravated liver injury, and exacerbation of cholangitis was considered. The initial UDCA dose was $600 \mathrm{mg}$ per day based on the Japanese guidelines. The patient's transaminase levels improved in conjunction with body weight loss, but the improvement in the $\gamma$-GTP level ceased once he reached a body weight of $92 \mathrm{~kg}$. The optimum dose in $\mathrm{PBC}$ patients is $13-15 \mathrm{mg} / \mathrm{kg}(3,8)$. UDCA changes the bile acid composition and reduces bile acid cytotoxicity in a concentration-dependent manner. In this case, the dose of UDCA may have been insufficient to ameliorate cholangitis. Furthermore, an increase in the UDCA dose in NASH patients may improve liver injury (19). Therefore, the UDCA dose was increased to $900 \mathrm{mg}$ per day, which is the maximum permissible dose in Japan. The patient's body weight was maintained at $92 \mathrm{~kg}$ for several months at this UDCA dose, and his $\gamma$-GTP level decreased. Therefore, an appropriate UDCA dose may improve the $\gamma$-GTP level in NASH patients with $\mathrm{PBC}$.

In conclusion, we herein described a case of NASH complicated with PBC. A detailed evaluation of the cause of liver injury is important for AMA-positive obese patients. Careful follow-up is required to monitor cholangitis after improvements in liver injury and fatty liver. A larger number of cases must be examined in order to clarify the pathogenesis of NASH accompanied by AMA positivity.

The authors state that they have no Conflict of Interest (COI).

\section{References}

1. Neuschwander-Tetri BA, Caldwell SH. Nonalcoholic steatohepatitis: summary of an AASLD Single Topic Conference. Hepatology 37: 1202-1219, 2003.

2. Bedossa P. Histological assessment of NAFLD. Dig Dis Sci 61: 1348-1355, 2016.

3. European Association for the Study of the liver. EASL clinical practice guidelines: the diagnosis and management of patients with primary biliary cholangitis. J Hepatol 67: 145-172, 2017.
4. Ravi S, Shoreibah M, Raff E, et al. Autoimmune markers do not impact clinical presentation or natural history of steatohepatitisrelated liver disease. Dig Dis Sci 60: 3788-3793, 2015.

5. Loria $\mathrm{P}$, Lonardo A, Leonardi F, et al. Non-organ-specific autoantibodies in nonalcoholic fatty liver disease: prevalence and correlates. Dig Dis Sci 48: 2173-2181, 2003.

6. Neuschwander-Tetri BA, Clark JM, Bass NM, et al. Clinical, laboratory and histological associations in adults with nonalcoholic fatty liver disease. Hepatology 52: 913-924, 2010.

7. Muratori P, Muratori L, Gershwin ME, et al. 'True' antimitochondrial antibody-negative primary biliary cirrhosis, low sensitivity of the routine assays, or both? Clin Exp Immunol 135: 154-158, 2004.

8. Corpechot C, Chretien Y, Chazouilleres O, Poupon R. Demographic, lifestyle, medical and familial factors associated with primary biliary cirrhosis. J Hepatol 53: 162-169, 2010.

9. Lindor KD, Gershwin ME, Poupon R, Kaplan M, Bergasa NV, Heathcote EJ. Primary biliary cirrhosis. Hepatology 50: 291-308, 2009.

10. Shibata M, Onozuka Y, Morizane T, et al. Prevalence of antimitochondrial antibody in Japanese corporate workers in Kanazawa prefecture. J Gastroenterol 39: 255-259, 2004.

11. Cacoub P, Saadoun D, Bourliere M, et al. Hepatitis B virus genotypes and extrahepatic manifestations. J Hepatol 43: 764-770, 2005.

12. Li BA, Liu J, Hou J, et al. Autoantibodies in Chinese patients with chronic hepatitis B: prevalence and clinical associations. World $\mathrm{J}$ Gastroenterol 21: 283-291, 2015.

13. Ramos-Casals M, Pares A, Jara LJ, et al. Antimitochondrial antibodies in patients with chronic hepatitis $\mathrm{C}$ virus infection: description of 18 cases and review of the literature. J Viral Hepat 12: 648-654, 2005

14. Nezu S, Tanaka A, Yasui H, et al. Presence of antimitochondrial autoantibodies in patients with autoimmune hepatitis. J Gastroenterol Hepatol 21: 1448-1454, 2006.

15. Leung PS, Rossaro L, Davis PA, et al. Antimitochondrial antibodies in acute liver failure: implication for primary biliary cirrhosis. Hepatology 46: 1436-1442, 2007.

16. Bogdanos DP, Baum H, Grasso A, et al. Microbial mimics are major targets of crossreactivity with human pyruvate dehydrogenase in primary biliary cirrhosis. J Hepatol 40: 31-39, 2004.

17. Rinella ME. Nonalcoholic fatty liver disease. A systemic review. JAMA 313: 2263-2273, 2015.

18. Chalasani N, Younossi Z, Lavine JE, et al. The diagnosis and management of non-alcoholic fatty liver disease: practice guideline by the American association for the study of liver disease, American college of gastroenterology, and the American gastroenterological association. Hepatology 55: 2005-2023, 2012.

19. Ratziu V, de Ledinghen V, Oberti F, et al. A randomized controlled trial of high dose ursodesoxycholic acid for nonalcoholic steatohepatitis. J Hepatol 54: 1011-1019, 2011.

The Internal Medicine is an Open Access journal distributed under the Creative Commons Attribution-NonCommercial-NoDerivatives 4.0 International License. To view the details of this license, please visit (https://creativecommons.org/licenses/ by-nc-nd/4.0/).

(C) 2018 The Japanese Society of Internal Medicine Intern Med 57: 3093-3097, 2018 